PHYSICAL REVIEW D 96, 039901(E) (2017)

\title{
Erratum: Threshold expansion of the three-particle quantization condition [Phys. Rev. D 93, 096006 (2016)]
}

\author{
Maxwell T. Hansen and Stephen R. Sharpe \\ (Received 5 July 2017; published 3 August 2017)
}

DOI: 10.1103/PhysRevD.96.039901

We have found an error in Eq. (126) of our paper, which leads to a change in the coefficient of the geometrical constant $\mathcal{K}$ in the final result for the threshold energy shift.

The left-hand side of Eq. (126) involves factors of

$$
\tilde{G}_{0 p}=\tilde{G}_{p 0}=\frac{1}{8 L^{3} m \omega_{p}^{2}} \frac{1}{E-m-2 \omega_{p}},
$$

evaluated at $E=3 m+\Delta E_{\mathrm{th}}$. The missed term occurs in the region where $p L \sim L^{0}$, for which we have

$$
\tilde{G}_{0 p}=-\frac{1}{8 m^{2} L}\left[\frac{1}{p^{2} L^{2}}+\frac{x}{L} \frac{1}{p^{4} L^{4}}+\mathcal{O}\left(L^{-2}\right)\right],
$$

where $x=\Delta E_{\mathrm{th}} L^{3} m^{2} \sim L^{0}$. The term proportional to $x$ in this equation was erroneously dropped in our paper. Including this term, the left-hand side (lhs) of Eq. (126) is augmented by the following term,

$$
9 \tilde{\mathcal{K}}_{2,00}^{2} \frac{2 \pi a}{m^{3}} \frac{\mathcal{K} x}{(2 \pi)^{6}}, \quad[\text { add to lhs of Eqs.(126), (127) and (135)], }
$$

where $\mathcal{K}=\sum_{\vec{n} \neq 0} 1 /\left(\vec{n}^{2}\right)^{3}$ is a numerical constant. This correction then propagates into the left-hand sides of Eqs. (127) and (135). This, in turn, leads to the following additional contribution to the right-hand side (rhs) of Eq. (135),

$$
\frac{576}{\pi^{2}} a^{4} m^{2} 24 \mathcal{K}, \quad \text { [add to rhs of Eq.(135)]. }
$$

This changes the coefficient of $\mathcal{K}$ on the rhs of Eq. (135) from -9 to +15 , and this change carries over to the final result of our paper, Eq. (136), which gives the coefficient of the $L^{-6}$ contribution to the threshold energy shift for three particles in a finite, cubic box. The corrected form of this equation reads

$$
\begin{aligned}
\frac{a_{6}(L)}{a_{3}}= & \left(\frac{a}{\pi}\right)^{3}\left[-\mathcal{I}^{3}+\mathcal{I} \mathcal{J}+15 \mathcal{K}+\frac{16 \pi^{3}}{3}(3 \sqrt{3}-4 \pi) \log \left(\frac{m L}{2 \pi}\right)+\mathcal{C}_{F}+\mathcal{C}_{4}+\mathcal{C}_{5}\right] \\
& +\frac{64 \pi^{2} a^{2}}{m} \mathcal{C}_{3}+\frac{3 \pi a}{m^{2}}+6 \pi r a^{2}-\frac{\mathcal{M}_{3, \text { thr }}}{48 m^{3} a_{3}} .
\end{aligned}
$$

We note that, with this change, the coefficient of $\mathcal{K}$ agrees with that obtained using nonrelativistic quantum mechanics (NRQM) in Ref. [1]. We had not previously been concerned about having different coefficients for $\mathcal{K}$ because, as shown in our paper, relativistic effects start to enter in $a_{6}(L)$. Furthermore, the two works used different ultraviolet regularization schemes, which could lead to differences in the finite terms. Nevertheless, it is interesting that the coefficient of $\mathcal{K}$ (like those of $\mathcal{I}^{3}$ and $\mathcal{I} \mathcal{J}$ ) do agree between the relativistic and NR calculations.

This error was discovered when performing a check of the result for $a_{6}(L)$ in $\lambda \phi^{4}$ theory. Previously, we had checked the contributions of third order in $\lambda$ (equivalent to third order in $a$ ) [2]; the check has now been extend to fourth order [3], verifying the corrected form in Eq. (5).

[1] S. R. Beane, W. Detmold, and M. J. Savage, Phys. Rev. D 76, 074507 (2007).

[2] M. T. Hansen and S. R. Sharpe, Phys. Rev. D 93, 014506 (2016).

[3] S. R. Sharpe, arXiv:1707.04279. 